\title{
Nonlinear pricing and type-dependent network effects
}

\author{
Arun Sundararajan* \\ Leonard N. Stern School of Business, New York University, 44 West 4th Street KMC 8-93, New York, NY 10012-1126, USA
}

Received 7 August 2003; accepted 29 October 2003

\begin{abstract}
Network effects can reduce consumption levels across customers when a monopolist chooses optimal nonlinear pricing. The direction and extent of these distortions depend on the relative rates of variation in marginal intrinsic value and marginal network value with customer type.

(C) 2004 Elsevier B.V. All rights reserved.
\end{abstract}

Keywords: Nonlinear pricing; Type-dependent; Network effects

JEL classification: D42; L12

\section{Introduction}

This paper analyzes optimal monopoly pricing under incomplete information for a good that displays positive network effects. In contrast with standard models of network effects (Katz and Shapiro, 1985), the good modeled in this paper is consumed in variable quantities by heterogeneous customers, and the magnitude of the network effects therefore depends on the total quantity consumed across customers, rather than the total number of adopters. In addition, the value each customer gets on account of the network effects depends on the customer's individual consumption, as well as the customer's type. Examples of products that fit this description at least partially include corporate desktop software (where customers are corporations of varying sizes, with varying intensity of software usage across employees) and online trading services (such as those offered by eBay, where network effects increase with increased trading volume).

* Tel.: +1-212-998-0833; fax: +1-212-995-4228.

E-mail address: asundara@stern.nyu.edu (A. Sundararajan). 


\section{Model}

A monopolist sells a homogeneous network good which may be used in continuously varying quantities. Fixed and variable production costs are assumed to be zero. Customers are heterogeneous, indexed by type $\theta \in[0,1]$ which has an absolutely continuous distribution function $F(\theta)$. The preferences of a customer of type $\theta$ are represented by $U(q, \theta, Q)-p$, where $q$ is the individual consumption of the customer, $Q$ is the gross consumption across all customers, and $p$ is total price.

For each $Q \geq 0, U(q, \theta, Q)$ has properties in $q$ and $\theta$ that are standard in nonlinear pricing problems: ${ }^{1}$ for finite $q, U_{1}(q, \theta, Q)>0, U_{11}(q, \theta, Q)<0, U_{12}(q, \theta, Q)>0$, and $\frac{\mathrm{d}}{\mathrm{d} \theta}\left(\frac{-U_{11}(q, \theta, Q)}{U_{1}(q, \theta, Q)}\right)<0$. $U(q, \theta, Q)$ is assumed to be bounded. The hazard rate of the distribution of $\theta$, denoted $H(\theta)=\frac{f(\theta)}{1-F(\theta)}$, is assumed nondecreasing. Additionally, $U(q, \theta, Q)$ can be separated into two components:

$$
U(q, \theta, Q)=v(q, \theta)+w(q, \theta, Q)
$$

where $v(q, \theta)$ is termed intrinsic value, and $w(q, \theta, Q)$ is termed network value. In the absence of network effects, it is assumed that $U(q, \theta, Q)=v(q, \theta)$, which allows a straightforward comparison of outcomes with those that obtain in the absence of network effects. The network effects are positive, so $w_{3}(q, \theta, Q) \geq 0$; in addition, $w(q, \theta, 0)=0$ for all $q, \theta$, which implies that $U(q, \theta, 0)=v(q, \theta)$, and $v(q, \theta)$, therefore, has all the properties ascribed to $U(q, \theta, Q)$ in its first two arguments.

Since $U_{1}(q, \theta, Q)>0$ for all finite $q$ and $U(q, \theta, Q)$ is bounded, $\lim _{q \rightarrow \infty} U_{1}(q, \theta, Q)=0$. The efficient consumption level is therefore infinite for all types.

\subsection{Feasible pricing}

Nonlinear pricing schedules (contracts) are represented by quantity-price pairs $q(\theta), \tau(\theta)$ indexed by $\theta \in[0,1]$. Given an expectation $Q$ of gross consumption that is shared by all customers, a contract $q(\theta), \tau(\theta)$ is said to be feasible for $Q$ if, for each $\theta$, it satisfies incentive compatibility and individual rationality:

$$
\begin{aligned}
& \theta \in \arg \max _{t} U(q(t), t, Q)-\tau(t) \\
& U(q(\theta), \theta, Q)-\tau(\theta) \geq 0 .
\end{aligned}
$$

A contract $q(\theta), \tau(\theta)$ satisfies fulfilled expectations (Radner, 1982) if it is feasible for $Q=\int_{0}^{1} q(\theta)$ $f(\theta) \mathrm{d} \theta$.

\subsection{Optimal pricing}

The monopolist seeks the profit-maximizing feasible contract that satisfies fulfilled expectations. This contract is denoted $q^{*}(\theta), \tau *(\theta)$. Fix an expectation $Q$ of gross consumption that is shared by all

\footnotetext{
${ }^{1}$ Numbered subscripts of functions refer to partial derivatives with respect to the corresponding variable.
} 
customers. Given $Q$, it follows from well-known nonlinear pricing results (Maskin and Riley, 1984) that the profit-maximizing feasible contract, denoted $q(\theta, Q), \tau(\theta, Q)$, will solve

$$
\begin{aligned}
& \frac{U_{12}(q(\theta, Q), \theta, Q)}{U_{1}(q(\theta, Q), \theta, Q)}=H(\theta) \\
& \tau(\theta, Q)=U(q(\theta, Q), \theta, Q)-\int_{0}^{\theta} U_{2}(q(t, Q), t, Q) \mathrm{d} t
\end{aligned}
$$

for each $\theta \in[0,1]$ and will be unique for a fixed, exogenously specified $Q$. Given any such $Q$, we define the actual consumption function

$$
\Gamma(Q)=\int_{0}^{1} q(\theta, Q) f(\theta) \mathrm{d} \theta
$$

where $q(\theta, Q)$ is defined according to Eq. (4). Combining profit maximization and the restriction of satisfying fulfilled expectations, it follows that the optimal contract satisfies Eqs. (4) and (5) for a value of $Q$ that is a fixed point of $\Gamma(Q)$. Using Eq. (1), this implies that $q *(\theta), \tau *(\theta)$ satisfies

$$
\begin{aligned}
& \frac{v_{12}\left(q^{*}(\theta), \theta\right)+w_{12}\left(q^{*}(\theta), \theta, Q^{*}\right)}{v_{1}\left(q^{*}(\theta), \theta\right)+w_{1}\left(q^{*}(\theta), \theta, Q^{*}\right)}=H(\theta) \\
& \tau^{*}(\theta)=v\left(q^{*}(\theta), \theta\right)+w\left(q^{*}(\theta), \theta, Q^{*}\right)-\int_{0}^{\theta}\left[v_{2}\left(q^{*}(t), t\right)+w_{2}\left(q^{*}(t), t, Q^{*}\right)\right] \mathrm{d} t,
\end{aligned}
$$

where $Q^{*} \int_{0}^{1} q^{*}(\theta) f(\theta) \mathrm{d} \theta$. There may be multiple contracts which satisfy these conditions, since the uniqueness of a fixed point of $\Gamma(Q)$ is not guaranteed. ${ }^{2}$ The existence of a fixed point of $\Gamma(Q)$ is discussed in Appendix A; the monopolist simply picks the one that is most profitable. Finally, we denote the optimal contract in the absence of network effects as $q^{\mathrm{o}}(\theta), \tau^{\mathrm{O}}(\theta)$. This contract is unique and specified by

$$
\begin{aligned}
& \frac{v_{12}\left(q^{\mathrm{o}}(\theta), \theta\right)}{v_{1}\left(q^{\mathrm{o}}(\theta), \theta\right)}=H(\theta) \\
& \tau^{\mathrm{o}}(\theta)=v\left(q^{\mathrm{o}}(\theta), \theta\right)-\int_{0}^{\theta} v_{2}\left(q^{\mathrm{o}}(t), t\right) \mathrm{d} t .
\end{aligned}
$$

For type $\theta=1$, both these contracts yield the efficient individual consumption level $\mathrm{q}=\infty$. Since $F(\theta)$ is absolutely continuous, $H(\theta)>0$ for $\theta<1$, and consumption is finite for types $\theta \in[0,1)$.

\footnotetext{
2 The existence of a fixed point of $\Gamma(Q)$ is discussed in Appendix A.
} 


\section{Comparison of outcomes and discussion}

One can now compare outcomes in the presence of network effects with those obtained in their absence. If one defines $Q^{\mathrm{o}}=\int_{0}^{1} q^{\mathrm{o}}(\theta) f(\theta) \mathrm{d} \theta$, then the contract

$$
\begin{aligned}
q(\theta) & =q^{\mathrm{o}}(\theta) \\
\tau(\theta) & =\tau^{\mathrm{o}}(\theta)+\left(w\left(q^{\mathrm{o}}(\theta), \theta, Q^{\mathrm{o}}\right)-\int_{\mathrm{o}}^{\theta} w_{2}\left(q^{\mathrm{o}}(t), t, Q^{\mathrm{o}}\right) \mathrm{d} t\right)
\end{aligned}
$$

is feasible, satisfies fulfilled expectations, and yields strictly higher profits in the presence of network effects than $q^{\mathrm{o}}(\theta), \tau^{\mathrm{o}}(\theta)$ does in their absence. Since $q^{*}(\theta), \tau *(\theta)$ yields profits at least as high as those from $q(\theta), \tau(\theta)$, the presence of network effects is always strictly profit improving for the monopolist. It is also straightforward to show that consumer surplus of type $\theta$ strictly increases if $q^{*}(\theta) \geq q^{\mathrm{O}}(\theta)$ and $Q^{*} \geq Q^{\mathrm{o}}$. If these conditions are not met, the direction of change in surplus depends on the interaction between individual consumption $q$ and gross consumption $Q$ in the function $w(q, \theta, Q)$.

Characterizing the direction of changes in consumption requires the following lemma:

Lemma 1. If $\frac{d}{d \theta}\left(\frac{-U_{11}(q, \theta, Q)}{U_{1}(q, \theta, Q)}\right)<0$, then $\frac{d}{d q}\left(\frac{U_{12}(q, \theta, Q)}{U_{1}(q, \theta, Q)}\right)>0$.

\section{Proof}

$$
\frac{\mathrm{d}}{\mathrm{d} \theta}\left(\frac{-U_{11}(q, \theta)}{U_{1}(q, \theta)}\right)=\frac{-U_{112}(q, \theta) U_{1}(q, \theta)+U_{11}(q, \theta) U_{12}(q, \theta)}{\left(U_{1}(q, \theta)\right)^{2}}
$$

and

$$
\frac{\mathrm{d}}{\mathrm{d} q}\left(\frac{U_{1}(q, \theta)}{U_{12}(q, \theta)}\right)=\frac{U_{11}(q, \theta) U_{12}(q, \theta)-U_{112}(q, \theta) U_{1}(q, \theta)}{\left(U_{12}(q, \theta)\right)^{2}}
$$

The denominators of the RHS of Eqs. (11) and (12) are both strictly positive, and their numerators are identical. Therefore, $\frac{\mathrm{d}}{\mathrm{d} q}\left(\frac{U_{1}(q, \theta)}{U_{12}(q, \theta)}\right)<0$, which implies that $\frac{\mathrm{d}}{\mathrm{d} q}\left(\frac{U_{12}(q, \theta)}{U_{1}(q, \theta)}\right)>0$.

Lemma 1 enables the comparison of individual consumption levels that are induced by the optimal contract $q *(\theta), \tau *(\theta)$ with those that occur in the absence of network effects:

Proposition 1. For each $\theta$ :

(a) If $\frac{v_{12}\left(q^{*}(\theta), \theta\right)}{v_{1}\left(q^{*}(\theta), \theta\right)}<\frac{w_{12}\left(q^{*}(\theta), \theta, Q^{*}\right)}{w_{1}\left(q^{*}(\theta), \theta, Q^{*}\right)}$, then $q^{*}(\theta)<q^{o}(\theta)$.

(b) If $\frac{v_{12}\left(q^{*}(\theta), \theta\right)}{v_{1}\left(q^{*}(\theta), \theta\right)}>\frac{w_{12}\left(q^{*}(\theta), \theta, Q^{*}\right)}{w_{1}\left(q^{*}(\theta), \theta, Q^{*}\right)}$, then $q^{*}(\theta)>q^{o}(\theta)$. 
Proof. Let $\frac{v_{12}\left(q^{*}(\theta), \theta\right)}{v_{1}\left(q^{*}(\theta), \theta\right)}<\frac{w_{12}\left(q^{*}(\theta), \theta, Q^{*}\right)}{w_{1}\left(q^{*}(\theta), \theta, Q^{*}\right)}$. Straightforward algebra ${ }^{3}$ yields

$$
\frac{v_{12}\left(q^{*}(\theta), \theta\right)+w_{12}\left(q^{*}(\theta), \theta, Q^{*}\right)}{v_{1}\left(q^{*}(\theta), \theta\right)+w_{1}\left(q^{*}(\theta), \theta, Q^{*}\right)}>\frac{v_{12}\left(q^{*}(\theta), \theta\right)}{v_{1}\left(q^{*}(\theta), \theta\right)} .
$$

Eqs. (7) and (13) yield

$$
\frac{v_{12}\left(q^{*}(\theta), \theta\right)}{v_{1}\left(q^{*}(\theta), \theta\right)}<H(\theta)
$$

Eqs. (9) and (14) yield

$$
\frac{v_{12}\left(q^{*}(\theta), \theta\right)}{v_{1}\left(q^{*}(\theta), \theta\right)}<\frac{v_{12}\left(q^{\mathrm{o}}(\theta), \theta\right)}{v_{1}\left(q^{\mathrm{o}}(\theta), \theta\right)} .
$$

Lemma 1 ensures that $\frac{\mathrm{d}}{\mathrm{d} q}\left(\frac{v_{12}(q, \theta)}{v_{1}(q, \theta)}\right)>0$, and part (a) follows. Similar steps yield part (b).

Immediate corollaries are that if $w(q, \theta, Q)$ is constant in $q$, then individual consumption is unchanged by network effects for all types, since $w(q, \theta, Q)=w_{12}(q, \theta, Q)=0$. Alternately, if network value is increasing in individual and gross consumption, but constant in $\theta$, then individual consumption increases for all but the highest type, since $w_{1}(q, \theta, Q)>0$, while $w_{12}(q, \theta, Q)=0$ at any $q, \theta, Q$, implying that $q^{*}(\theta)>q^{0}(\theta)$ for $\theta \in[0,1)$. Profits and consumer surplus increase as well, and this might be expected as a 'normal' consequence of the presence of positive network effects. Both these cases are characterized in some detail in Sundararajan (2003).

However, the general case admits a wider variety of outcomes. Proposition 1 makes sense intuitively if one recognizes $\frac{v_{12}(q, \theta)}{v_{1}(q, \theta)}$ as the percentage change in marginal intrinsic value that results from a marginal increase in type, and $\frac{w_{12}(q, \theta, Q)}{w_{1}(q, \theta, Q)}$ as the percentage change in marginal network value for a marginal increase in type. Consider, for instance, the case when $\frac{w_{12}(q, \theta, Q)}{w_{1}(q, \theta, Q)}>\frac{v_{12}(q, \theta)}{v_{1}(q, \theta)}$ for all $q<\infty, \theta \in[0,1]$, and at all values of $Q$. In this case, the network effects increase the marginal impact of an increase in type on total marginal value (or loosely, makes the customer types more different from each other). Consequently, the monopolist changes pricing in a manner that increases the differences in consumption across types, which reduces consumption for all but the highest type. Accordingly, Proposition 1(a) concludes that $q *(\theta)<q^{0}(\theta)$ for $\theta \in[0,1)$. This distortion occurs despite the fact that the positive network effects increase value from consumption for all types, at all consumption levels. Any increase in consumption for a subset of types would increase the total surplus generated by all types, but at the cost of reducing the monopolist's ability to price discriminate. This case therefore highlights socially suboptimal trade-offs that a price-discriminating monopolist who controls a network good can make between value creation and price discrimination.

The reduction in individual consumption across all types described above is because efficient individual consumption is infinite for the highest type (or more importantly, because efficient individual consumption levels are unaltered by the network effects). In contrast, if the efficient level for the highest

\footnotetext{
${ }^{3}$ For $a, b, c, d>0, \frac{a}{b}<\frac{c}{d} \Rightarrow \mathrm{bc}-\mathrm{ad}>0$. Therefore, $\frac{a+c}{b+d}-\frac{a}{b}=\frac{b c-a d}{b(b+d)}>0$, or $\frac{a+c}{b+d}>\frac{a}{b}$.
} 
type strictly increased on account of the network effects, this would ensure an increase in consumption for a subset (of positive measure) of types. Under the condition $\frac{w_{12}(q, \theta, Q)}{w_{1}(q, \theta, Q)}>\frac{v_{12}(q, \theta)}{v_{1}(q, \theta)}$, this subset would be an interval of higher types; the reduction in individual consumption would be induced for the remaining interval of (lower) types, so long as the increase in efficient levels was highest for $\theta=1$. Network effects of this kind therefore increase consumption and surplus disparities across types, and while benefiting higher customer types, may have a net negative effect on the other (lower type) customers.

\section{Conclusion}

Network effects always increase monopoly profits. However, their presence can cause a pricediscriminating monopolist to distort individual consumption in a manner that reduces consumption for a fraction of (and sometimes all) customer types, and this may adversely affect the magnitude and distribution of consumer surplus. Ongoing work aims to refine the characterization of changes in consumption by placing more structure on the network value function $w(q, \theta, Q)$, and by exploiting properties of the optimal pricing schedule. Solving a multidimensional model which allows changes in individual and network value to vary with independent dimensions of type is also work in progress.

\section{Acknowledgements}

I thank Luis Cabral, Nicholas Economides, Roy Radner, participants at the 2003 meeting of the Society for the Advancement of Economic Theory and seminar participants at New York University for their comments. The usual disclaimers apply.

\section{Appendix A. Existence of a nontrivial fixed point of $\Gamma(Q)$}

Since $U(q, \theta, 0)=v(q, \theta)$, it follows that $\Gamma(0)=Q^{\mathrm{o}}$, where $Q^{o}=\int_{0}^{1} q^{\mathrm{o}}(\theta) f(\theta) \mathrm{d} \theta$. Therefore, $\Gamma(0)>0$. As a consequence, if $\Gamma(Q)$ is bounded, a fixed point exists at some $Q>0$. Recall the definition of $\Gamma(Q)$

$$
\Gamma(Q)=\int_{0}^{1} q(\theta, Q) f(\theta) \mathrm{d} \theta
$$

and define

$$
\bar{q}(\theta)=\lim _{Q \rightarrow \infty} q(\theta, Q)
$$

Since $F(\theta)$ is absolutely continuous, any condition that ensures the finiteness of $\bar{q}(\theta)$ for all $\theta<1$ will ensure that $\lim _{Q \rightarrow \infty} \Gamma(Q)$ is finite.

Suppose the sequence of functions $U(q, \theta, Q)$ indexed by $Q$ converges uniformly to a limit $\bar{u}(q, \theta)$ as $Q \rightarrow \infty$. It follows that

$$
\lim _{Q \rightarrow \infty} U_{1}(q, \theta, Q)=\bar{u}_{1}(q, \theta)
$$


Since $U_{1}(q, \theta, Q)$ is continuous, so is $\bar{u}_{1}(q, \theta)$. We already know that $\lim _{q \rightarrow \infty} U_{1}(q, \theta, Q)=0$ for each $Q$, and therefore, $\lim _{q \rightarrow \infty} \bar{u}_{1}(q, \theta)=0$. As a result, as long as $\bar{q}(\theta)$ is well defined, it is finite for all $\theta<1$, since Eq. (4) ensures that $\bar{u}_{1}(\bar{q}(\theta), \theta)>0$.

\section{References}

Katz, M., Shapiro, C., 1990. Network externalities, competition and contracting. American Economic Review 75, 424-440. Maskin, E., Riley, J., 1984. Monopoly with incomplete information. Rand Journal of Economics 15, $171-196$.

Radner, R., 1982. Equilibrium Under Uncertainty. In: Arrow, K., Intriligator, M. (Eds.), Handbook of Mathematical Economics. North Holland, Amsterdam, pp. 923-1006.

Sundararajan, A., 2003, Network Effects, Nonlinear Pricing and Entry Deterrence, available at http://ssrn.com/abstract= 382962. 